\title{
Numerical Identification of a Spatially Varying Diffusion Coefficient
}

\author{
By Gerard R. Richter
}

\begin{abstract}
We consider the inverse problem of identifying a spatially varying diffusion coefficient on the basis of an observed solution to the forward problem. Under appropriate conditions, this inverse problem can be solved as a first order hyperbolic problem in the unknown coefficient. We provide a modified upwind difference scheme for this hyperbolic problem and prove that its convergence rate is $O(h)$ when certain conditions are met.
\end{abstract}

1. Introduction. The partial differential equation

$$
\beta \frac{\partial u}{\partial t}=\nabla \cdot \alpha \nabla u+q
$$

is a basic governing equation in the analysis of aquifers (Bear [1]). The coefficients $\alpha$ and $\beta$ are often taken as functions of the space variable $x$, and their identification, using observed $u$ and $q$ data, constitutes an important inverse problem in groundwater flow and oil reservoir simulation.

We shall be concerned with the identification of the "transmissivity" $\alpha$ from observations taken under steady state conditions. The inverse problem then becomes a linear first order hyperbolic equation in $\alpha$ :

$$
L(\alpha ; u) \equiv \nabla \alpha \cdot \nabla u+\alpha \Delta u=f, \quad x \in \Omega,
$$

where $f=-q$. Assuming appropriate Cauchy data for $\alpha$ is available, and that the measurements are sufficiently definitive to permit approximation of $\nabla u, \Delta u$, and $f$ in $\Omega$, one may attempt to identify $\alpha$ by solving (1) numerically. This hyperbolic problem has several distinguishing features: (i) it need not have a timelike direction (i.e., a fixed direction in which the tangent vector along characteristics always has a positive component), (ii) its coefficients are derivatives of the observed quantity $u$ (a source of instability in practice), (iii) its first order term vanishes at extreme points of $u$.

Several alternatives for the numerical solution of (1) have been proposed in the applied literature. Nelson [5] has devised a method for integrating the homogeneous version of (1) (i.e., $f=0$ ) along its characteristics, and Frind and Pinder [3] have developed a finite element Galerkin technique. A finite difference approach has also been proposed by Nutbrown [6].

In a companion paper [7], dealing with some mathematical aspects of the inverse problem, we showed that (1) may be solved uniquely for $\alpha$ assuming

$$
\inf _{\Omega}\{\max [|\nabla u|, \Delta u]\}>0
$$

Received October 31, 1978; revised October 18, 1979 and August 21, 1980.

1980 Mathematics Subject Classification. Primary 65M05, 65M10.

() 1981 American Mathematical Society 0025-5718/81/0000-0052/\$04.00 
and that Cauchy data is available along the "inflow" portion of the boundary $\Gamma$ of $\Omega$ (essentially that portion of $\Gamma$ where the outer normal derivative of $u$ is negative). If (2) is not satisfied (which will be the case, for example, if $u$ has both maxima and minima in $\Omega$ ), then the hyperbolic problem may not be solvable for all $f$. We also considered the following "test conditions" for observing a "forward" solution to the elliptic problem for (1):

$$
\operatorname{Inf}_{\Omega} f>0, \quad u=\text { constant on } \Gamma .
$$

Assuming the unknown $\alpha$ is positive, the resulting $u$ then satisfies (2), and the corresponding hyperbolic problem (1) requires no Cauchy data for $\alpha$.

In the present paper, we present a modified upwind difference scheme which closely mimics the essential features of the continuous problem. The scheme is explicit in the direction of increasing grid values of $u$ (the characteristics of (1) are curves of steepest ascent in $u$ ), and it is self-starting in the vicinity of a relative minimum of $u$ (where (1) is degenerate and $\alpha$ is given by $f / \Delta u$ if $\Delta u \neq 0$ ). In Sections 2 and 3, we describe the numerical method for the special case where $\Omega$ is a unit square and establish its $O(h)$ convergence rate under the basic condition (2). It appears that the only other proof of convergence of a numerical scheme for (1) is that of Falk [2], which is based on a quite different nonlinear least squares approach. In Section 4, we extend the applicability of our numerical method and accompanying analysis to irregular domains. Then in Section 5, we show that the numerical method performs optimally under the "test conditions" alluded to in the previous paragraph. Finally, in Section 6, we present computational results for two test problems.

2. The Discrete Problem. We shall develop our numerical method in the context of a two-dimensional problem in the unit square $\Omega=(0,1) \times(0,1)$. Its extension to more general domains will be seen in Section 4 to be quite straightforward.

Our method utilizes a uniform grid

$$
\left(x_{i}, y_{j}\right)=(i h, j h), \quad 0<i, j<n+1, h=\frac{1}{n+1} .
$$

The set of interior grid points will be denoted by $\Omega^{h}$,

$$
\Omega^{h}=\left\{\left(x_{i}, y_{j}\right) \mid 1<i, j<n\right\}
$$

and for a grid quantity $\left\{v_{i j}\right\}$ defined over $\Omega^{h}$ we define

$$
\left\|v_{i j}\right\|_{\infty}=\max _{1<i, j<n}\left|v_{i j}\right| \text {. }
$$

We also define a discrete inflow boundary $\Gamma_{1}^{h}$. A grid point in $\Gamma$ is a member of $\Gamma_{1}^{h}$ if its nearest neighboring grid point in $\Omega^{h}$ has a higher $u$ value; e.g., $\left(x_{i}, y_{0}\right) \in \Gamma_{1}^{h}$ for $i \in\{1, \ldots, n\}$ if $u\left(x_{i}, y_{1}\right)>u\left(x_{i}, y_{0}\right)$. Henceforth, we shall denote grid values of $u(x, y)$ and $f(x, y)$ by $u_{i j}$ and $f_{i j}$, respectively.

We approximate the differential equation (1) by $\mathcal{L}^{h}\left\{\alpha_{i j} ; u_{i j}\right\}=f_{i j}, 1<i, j<n$, where

$$
\begin{gathered}
\mathcal{L}^{h}\left\{\alpha_{i j} ; u_{i j}\right\} \equiv \frac{\alpha_{i j}-\alpha_{k j}}{h} \cdot \frac{u_{i j}-u_{k j}}{h}+\frac{\alpha_{i j}-\alpha_{i l}}{h} \cdot \frac{u_{i j}-u_{i l}}{h}+\alpha_{i j} H u_{i j}, \\
H u_{i j} \equiv \frac{u_{i+1, j}+u_{i-1, j}+u_{i, j+1}+u_{i j-1}-4 u_{i j}}{h^{2}}
\end{gathered}
$$


$k$ is the first index of the minimum of $\left\{u_{i-1, j}, u_{i j}, u_{i+1, j}\right\}$,

$l$ is the second index of the minimum of $\left\{u_{i, j-1}, u_{i j}, u_{i, j+1}\right\}$.

Thus, $k$ and $l$ are in fact functions of the indices $i$ and $j$, which we do not make explicit for the sake of notational simplicity. In the event of a tie for $k$ or $l$, its resolution will be seen as immaterial in what follows.

Solving the equation $\mathcal{L}^{h}\left\{\alpha_{i j} ; u_{i j}\right\}=f_{i j}$ for $\alpha_{i j}$, we obtain

$$
\alpha_{i j}=\frac{\alpha_{k j}\left(\frac{u_{i j}-u_{k j}}{h}\right)+\alpha_{i l}\left(\frac{u_{i j}-u_{i l}}{h}\right)+h f_{i j}}{\frac{u_{i j}-u_{k j}}{h}+\frac{u_{i j}-u_{i l}}{h}+h H u_{i j}},
$$

which serves as the basis for several observations:

(i) The difference scheme is explicit in the direction of increasing $u_{i j}$ since $\alpha_{i j}$ is given in terms of $\alpha_{k j}$ and $\alpha_{i l}$, where $u_{k j}$ and $u_{i l}$ are $<u_{i j}$. Thus, the discrete solution is developed in a manner consistent with the characteristics of the continuous problem, which are curves of steepest ascent in $u$.

(ii) If $u_{i^{*}, j^{*}}$ is a relative minimum grid value of $u$, the associated $\alpha_{i j}$ is given by

$$
\alpha_{i^{*}, j^{*}}=\frac{f_{i^{*}, j^{*}}}{H u_{i^{*}, j^{*}}} .
$$

This corresponds to the case of degeneracy in the continuous problem, $\nabla \alpha \cdot \nabla u+$ $\alpha \Delta u=f$, where if $\Delta u(P)=0$ and $\Delta u(P) \neq 0$, then

$$
\alpha(P)=\frac{f(P)}{\Delta u(P)} \text {. }
$$

(iii) Initial data for $\left\{\alpha_{i j}\right\}$ is required along $\Gamma_{1}^{h}$.

(iv) The difference scheme is first order in $h$. Assuming $\alpha \in C^{2}(\bar{\Omega}), u \in C^{3}(\bar{\Omega})$, the accuracy of the difference approximations to the various terms of $L(\alpha ; u)=f$ is as follows:

\begin{tabular}{r|l} 
Term & bound on error in corresponding difference approximation \\
\hline$\left(\alpha_{x} u_{x}\right)\left(x_{i}, y_{j}\right)$ & $\frac{h}{2}\left[\left\|\alpha_{x}\right\|_{\infty} \cdot\left\|u_{x x}\right\|_{\infty}+|k-i|\left\{\left\|\alpha_{x x}\right\|_{\infty} \cdot\left\|u_{x}\right\|_{\infty}+\frac{h}{2}\left\|\alpha_{x x}\right\|_{\infty} \cdot\left\|u_{x x}\right\|_{\infty}\right\}\right]$ \\
$\left(\alpha_{y} u_{y}\right)\left(x_{i}, y_{j}\right)$ & $\frac{h}{2}\left[\left\|\alpha_{y}\right\|_{\infty} \cdot\left\|u_{y y}\right\|_{\infty}+|l-j| \cdot\left\{\left\|\alpha_{y y}\right\|_{\infty} \cdot\left\|u_{y}\right\|_{\infty}+\frac{h}{2}\left\|\alpha_{y y}\right\|_{\infty} \cdot\left\|u_{y y}\right\|_{\infty}\right\}\right]$ \\
$\alpha\left(u_{x x}+y_{y y}\right)\left(x_{i}, y_{j}\right)$ & $\frac{h}{3}\|\alpha\|_{\infty}\left[\left\|u_{x x x}\right\|_{\infty}+\left\|u_{y y y}\right\|_{\infty}\right]$
\end{tabular}

3. Analysis of the Difference Scheme. In a previous paper [7], we considered the hyperbolic differential equation (1) under the basic condition (2). This condition guarantees the existence of a unique solution $\alpha$ assuming prescribed values along the inflow portion of $\Gamma$, and can be realized physically by observing $u$ in the presence of a uniformly positive forcing function $f$. A condition equivalent to (2) is that the domain $\Omega$ can be divided into subregions $\Omega_{1}$ and $\Omega_{2}$ in which $|\nabla u|$ and $\Delta u$, respectively, are uniformly positive:

$$
\begin{aligned}
& \Omega=\Omega_{1} \cup \Omega_{2}, \\
& |\nabla u|>k_{1}>0 \text { in } \Omega_{1}, \\
& \Delta u>k_{2}>0 \text { in } \Omega_{2} .
\end{aligned}
$$


Our objective in this section is to establish the stability and convergence of the difference scheme for the unit square,

$$
\mathcal{L}^{h}\left\{\alpha_{i j} ; u_{i j}\right\}=f_{i j}, \quad 1<i, j<n, \alpha_{i j} \text { given on } \Gamma_{1}^{h},
$$

under the assumption that (9) holds. Our analysis will be seen in Section 4 to be readily extendable to irregular domains.

We begin the analysis by postulating a discrete analog of (9). We assume that the interior grid points $\Omega^{h}$ can be divided into two sets $\Omega_{1}^{h}$ and $\Omega_{2}^{h}$ such that

$$
\begin{aligned}
& \Omega^{h}=\Omega_{1}^{h} \cup \Omega_{2}^{h}, \\
& \max \left\{\frac{u_{i j}-u_{k j}}{h}, \frac{u_{i j}-u_{i l}}{h}\right\} \geqslant k_{1}^{h}>0, \quad\left(x_{i}, y_{j}\right) \in \Omega_{1}^{h}, \\
& H u_{i j} \geqslant k_{2}^{h}>0, \quad\left(x_{i}, y_{j}\right) \in \Omega_{2}^{h} .
\end{aligned}
$$

We also define

$$
q_{1}^{h}=\max _{\left(x_{i}, y_{j}\right) \in \Omega_{1}^{h}}\left\{\frac{-H u_{i j}}{\max \left\{\frac{u_{i j}-u_{k j}}{h}, \frac{u_{i j}-u_{i l}}{h}\right\}}\right\}
$$

which may be regarded as a discrete analog of the quantity

$$
q_{1}=\sup _{\Omega_{1}}\left\{\frac{-\Delta u}{|\nabla u|}\right\}
$$

Note that just as (9) rules out the possibility of $u$ having a relative maximum in $\Omega$, (11) precludes the possibility of a relative maximum $u_{i j}$ in $\Omega^{h}$ (i.e., one which is $>$ its four nearest neighbors). We isolate those interior grid points, if any, for which the corresponding $u_{i j}$ is a relative minimum and denote the resulting subset of $\Omega_{2}^{h}$ by $\Omega_{*}^{h}$.

At a point $\left(x_{i^{*}}, y_{j^{*}}\right) \in \Omega_{*}^{h}$, the associated $\alpha$ value is given explicitly by (7). For any other $\left(x_{i}, y_{j}\right) \in \Omega^{h}$, the value of $\alpha_{i j}$ is given in terms of one or two neighboring values, $\alpha_{k j}$ and/or $\alpha_{i l}$, associated with lower $u$ values. We depict the local dependence of such $\alpha_{i j}$ by means of arrows pointing along the corresponding segments from $\left(x_{k}, y_{j}\right)$ and/or $\left(x_{i}, y_{l}\right)$ to $\left(x_{i}, y_{j}\right)$. A path like one of those shown in Figure 1 which originates at $\Gamma_{1}^{h}$ or $\Omega_{*}^{h}$ and proceeds through $\Omega^{h}$ in accordance with the resulting network of arrows will be termed an "admissible path".

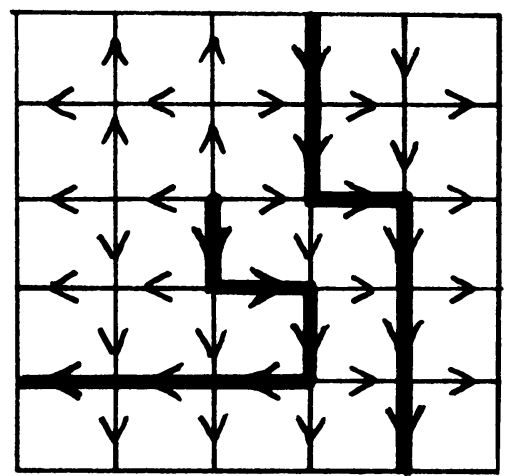

FIGURE 1

Admissible paths 
We observe that $u$ is nondecreasing along an admissible path, and that any point $\left(x_{i}, y_{j}\right) \in \Omega^{h}-\Omega_{*}^{h}$ can be connected to $\Gamma_{1}^{h}$ or $\Omega_{*}^{h}$ by at least one admissible path. Moreover, the set of all admissible paths to a given interior grid point describes the domain of dependence of the corresponding $\alpha_{i j}$.

We shall say that a segment of an admissible path from $\left(x_{k}, y_{j}\right)$ or $\left(x_{i}, y_{l}\right)$ to $\left(x_{i}, y_{j}\right) \in \Omega_{1}^{h}$ is "aligned" if it makes an angle $\pi / 4$ or less with the vector

$$
\nabla^{h} u_{i j}=\left(\frac{u_{i j}-u_{k j}}{h}, \frac{u_{i j}-u_{i l}}{h}\right) \text {. }
$$

Thus, a segment from $\left(x_{k}, y_{j}\right)$, for example, to $\left(x_{i}, y_{j}\right)$ is aligned if and only if $u_{k j} \leqslant u_{i l}$. Note that $u$ increases by at least $h k_{1}^{h}$ over each aligned segment of an admissible path, a key element in our subsequent analysis.

We now establish a series of lemmas which will lead eventually to a proof of stability and convergence of the numerical solution under the conditions described in (11).

LEMMA 1. If $a, b, c, d$ are scalars and $c, d$ have the same sign,

$$
\left|\frac{a+b}{c+d}\right| \leqslant \max \left\{\left|\frac{a}{c}\right|,\left|\frac{b}{d}\right|\right\}
$$

Proof. Suppose $\max \{|a / c|,|b / d|\}=|a / c|$. Then $|b|<|d| \cdot|a / c|$ and $|(a+b) /(c+d)| \leqslant(|a|+|b|) /|c+d| \leqslant(|a|+|d| \cdot|a / c|) /(|c|+|d|)=|a / c|$.

Lemma 2. If $\mathscr{P} \geqslant 0, \Re \geqslant 0, A_{0} \geqslant 0$, and $A_{i}=\mathscr{P}\left(A_{i-1}+\Re\right), i=1,2, \ldots$, then $A_{i}<\mathscr{P}^{i}\left(A_{0}+i \mathfrak{N}\right)$.

Proof. By induction on $i$.

LEMMA 3. Assuming (11) holds and $h q_{1}^{h}<1$, the discrete equations (10) have a unique solution $\left\{\alpha_{i j}\right\}$.

Proof. Since the scheme is explicit, we need only show that the denominator $D_{i j}$ in (6) is invariably nonzero. If $\left(x_{i}, y_{j}\right) \in \Omega_{2}^{h}$, then $D_{i j}>h k_{2}^{h}>0$ by (11). Next, suppose $\left(x_{i}, y_{j}\right) \in \Omega_{1}^{h}$ and consider the corresponding aligned segment to $\left(x_{i}, y_{j}\right)$. In the event $u_{k j} \leqslant u_{i l}$, this segment originates at $\left(x_{k}, y_{j}\right)$ so that $u_{i j}-u_{k j}>h k_{1}^{h}$ and

$$
D_{i j} \geqslant \frac{u_{i j}-u_{k j}}{h}\left[1+\frac{h H u_{i j}}{\left(\left(u_{i j}-u_{k j}\right) / h\right)}\right] \geqslant k_{1}^{h}\left(1-h q_{1}^{h}\right)>0 .
$$

The same bound also holds in the case $u_{i l}<u_{k j}$. Hence, $D_{i j}>0$ for all $i, j \in$ $\{1, \ldots, n\}$, and the discrete solution is well defined.

LEMMA 4. If $\left(x_{i}, y_{j}\right) \in \Omega_{2}^{h}$, then

$$
\left|\alpha_{i j}\right| \leqslant \max \left\{\left|\alpha_{k j}\right|,\left|\alpha_{i l}\right|, \frac{\|f\|_{\infty}}{k_{2}^{h}}\right\}
$$

where, if $k=i$ or $l=j$ or both, the corresponding quantities $\left|\alpha_{k j}\right|,\left|\alpha_{i l}\right|$ are deleted.

Proof. If $k=i$ and $l=j, \alpha_{i j}$ is given by (7) and does not exceed $\|f\|_{\infty} / k_{2}^{h}$ in absolute value. Assuming $k \neq i$ and/or $l \neq j$, we apply Lemma 1 to (6) with 
$b=h f_{i j}, d=h H u_{i j}$. This gives

$$
\left|\alpha_{i j}\right|<\max \left\{\frac{\left|\alpha_{k j}\right|\left(\frac{u_{i j}-u_{k j}}{h}\right)+\left|\alpha_{i l}\right|\left(\frac{u_{i j}-u_{i l}}{h}\right)}{\left(\frac{u_{i j}-u_{k j}}{h}\right)+\left(\frac{u_{i j}-u_{i l}}{h}\right)}, \frac{f_{i j}}{H u_{i j}}\right\},
$$

from which the desired result follows.

LEMMA 5. Assuming $h q_{1}^{h}<1$, let $\rho=1 /\left(1-h q_{1}^{h}\right)$ and $\eta=h\|f\|_{\infty} / k_{1}^{h}$. Then, if $\left(x_{i}, y_{j}\right) \in \Omega_{1}^{h}$,

$$
\left|\alpha_{i j}\right|< \begin{cases}\max \left\{\left|\alpha_{i l}\right|, \rho\left(\left|\alpha_{k j}\right|+\eta\right)\right\}, & u_{k j}<u_{i l} \\ \max \left\{\left|\alpha_{k j}\right|, \rho\left(\left|\alpha_{i l}\right|+\eta\right)\right\}, & u_{i l}<u_{k j}\end{cases}
$$

where, if $k=i$ or $l=j$, the corresponding $\left|\alpha_{k j}\right|$ or $\left|\alpha_{i l}\right|$ is deleted.

Proof. Again apply Lemma 1 to (6), this time with

$$
\begin{aligned}
& a=\alpha_{i l}\left(\frac{u_{i j}-u_{i l}}{h}\right) \text { and } c=\frac{u_{i j}-u_{i l}}{h} \quad \text { if } u_{k j}<u_{i l}, \quad \text { or } \\
& a=\alpha_{k j}\left(\frac{u_{i j}-u_{k j}}{h}\right) \text { and } c=\frac{u_{i j}-u_{k j}}{h} \quad \text { if } u_{i l}<u_{k j} .
\end{aligned}
$$

The desired result follows directly.

TheOREM 1. Assuming (11) holds and $h q_{1}^{h}<1$, the discrete problem (10) has a unique solution $\left\{\alpha_{i j}\right\}$ satisfying

$$
\left\|\alpha_{i j}\right\|_{\infty} \leqslant C_{1}^{h}(u) \cdot\left[\max \left\{\max _{\Gamma_{1}^{h}}\left|\alpha_{i j}\right|, \frac{\|f\|_{\infty}}{k_{2}^{h}}\right\}+\frac{[u]\|f\|_{\infty}}{\left(k_{1}^{h}\right)^{2}}\right],
$$

where

$$
C_{1}^{h}(u) \equiv \max \{1, \rho\}^{[u] / h k_{1}^{h}}, \quad[u] \equiv \sup _{\Omega} u-\inf _{\Omega} u .
$$

Proof. What remains to be shown is the bound on $\left\|\alpha_{i j}\right\|_{\infty}$. To obtain it, we define a new array $\left\{A_{i j}\right\}$ as follows:

$$
\begin{aligned}
& A_{i j}= \begin{cases}\max \left\{A_{i l}, \rho\left(A_{k j}+\eta\right)\right\}, & \left(x_{i}, y_{j}\right) \in \Omega_{1}^{h}, u_{k j}<u_{i l} \\
\max \left\{A_{k j}, \rho\left(A_{i l}+\eta\right)\right\}, & \left(x_{i}, y_{j}\right) \in \Omega_{1}^{h}, u_{i l}<u_{k j} \\
\max \left\{A_{k j}, A_{i l}\right\}, \quad\left(x_{i}, y_{j}\right) \in \Omega_{2}^{h}-\Omega_{*}^{h}\end{cases} \\
& A_{i j}=\max \left\{\max _{\Gamma_{1}^{h}}\left|\alpha_{i, j}\right|, \frac{\|f\|_{\infty}}{k_{2}^{h}}\right\}, \quad\left(x_{i}, y_{j}\right) \in \Gamma_{1}^{h} \cup \Omega_{*}^{h} .
\end{aligned}
$$

It is easy to see from Lemmas 4 and 5 that $A_{i j} \geqslant\left|\alpha_{i j}\right|$ for $\left(x_{i}, y_{j}\right) \in \Omega^{h} \cup \Gamma_{1}^{h}$. We will now show that

$$
A_{i j} \leqslant \max \left\{1, \rho^{m_{i j}}\right\} \cdot\left[\max \left\{\max _{\Gamma_{1}^{h}}\left|\alpha_{i j}\right|, \frac{\|f\|_{\infty}}{k_{2}^{h}}\right\}+m_{i j} \eta\right],
$$

where $m_{i j}$ is the maximum possible number of aligned segments in any admissible path from $\Gamma_{1}^{h} \cup \Omega_{*}^{h}$ to $\left(x_{i}, y_{j}\right)$. 
To see that (17) is valid, we choose an arbitrary pair of indices $i_{0}, j_{0} \in$ $\{1,2, \ldots, n\}$ and consider the corresponding quantity $A_{i_{0, j}}$. Assuming $\left(x_{i_{0}}, y_{j_{0}}\right) \notin$ $\Omega_{*}^{h}$ (otherwise (17) holds trivially), $A_{i_{0} j_{0}}$ is given in terms of a neighboring value $A_{i_{1} j_{1}}$ as

$$
\rho\left(A_{i_{1} j_{1}}+\eta\right) \text { or } A_{i_{1} j_{1}},
$$

depending on whether or not the segment from $\left(x_{i_{1}}, y_{j_{1}}\right)$ to $\left(x_{i_{0}}, y_{j_{0}}\right)$ is aligned. Likewise, if $\left(x_{i_{1}}, y_{j_{1}}\right) \in \Gamma_{1}^{h} \cup \Omega_{*}^{h}, A_{i_{1} j_{1}}$ is given in terms of a neighboring value $A_{i_{2} j_{2}}$. Continuing in this way we may trace the value of $A_{i_{0 j_{0}}}$ back to some $A_{i, j_{p}}$ where $\left(x_{i_{p}}, y_{j_{p}}\right) \in \Gamma_{1}^{h} \cup \Omega_{*}^{h}$. Note that the chain $\left(x_{i_{p}}, y_{j_{p}}\right), \ldots,\left(x_{i_{0}}, y_{j_{0}}\right)$ corresponds to an admissible path through $\Omega^{h}$. Along each segment of this admissible path, the value of $A_{i_{k} j_{k}}$ either changes to $\rho\left(A_{i_{k} j_{k}}+\eta\right)<\max \{1, \rho\} \cdot\left(A_{i_{k} j_{k}}+\eta\right)$ or remains the same, depending on whether the segment is aligned or not. Applying Lemma 2 over all possible paths from $\Gamma_{1}^{h} \cup \Omega_{*}^{h}$ to $\left(x_{i_{0}}, y_{j_{0}}\right)$, we obtain (17).

To complete the proof, we note that $m_{i j}<[u] / h k_{1}^{h}$. This follows from the fact that an admissible path is one of increasing $u$, and each of its aligned segments contributes an increase of at least $h k_{1}^{h}$. Combining the bound on $m_{i j}$ with (17), we obtain the desired result.

The theorem we have just proved is predicated on the assumption that $\left\{u_{i j}\right\}$ satisfies the discrete condition (11). We wish now to recast the theorem in terms of the analogous conditions (9) for $u(x, y)$, under which we shall establish the convergence of the numerical solution $\left\{\alpha_{i j}\right\}$. One additional definition is required:

$$
C_{1}(u) \equiv \max \left\{1, \exp \left(\frac{q_{1}[u]}{k_{1}}\right)\right\}
$$

We relate $k_{1}^{h}, k_{2}^{h}, q_{1}^{h}$ and $C_{1}^{h}(u)$ to their continuous counterparts as follows:

LEMMA 6. If (1) holds and $u \in C^{2}(\bar{\Omega})$, then (11) is satisfied by $\left\{u_{i j}\right\}$ for $h$ sufficiently small with

(i) $k_{1}^{h}>\left[k_{1}-(h / 2) \max \left\{\left\|u_{x x}\right\|_{\infty},\left\|u_{y y}\right\|_{\infty}\right\}\right] / \sqrt{2}$.

(ii) $k_{2}^{h}>k_{2}-o(h)$.

Furthermore,

(iii) If $q_{1}<0, q_{1}^{h}<0$ for $h$ sufficiently small. If $q_{1}>0, q_{1}^{h}<\sqrt{2} q_{1}[1+o(h)]$.

(iv) $C_{1}^{h}(u)<\left[C_{1}(u)^{2}\right] \cdot[1+o(h)]$.

Proof. (i) This follows from the formula

$$
\frac{u_{i j}-u_{k j}}{h}=\left|u_{x}\right|\left(x_{i}, y_{j}\right) \pm \frac{h}{2} u_{x x}\left(\xi_{i}, y_{j}\right), \quad \xi_{i} \in\left(x_{i-1}, x_{i+1}\right),
$$

the analogous formula for $\left(u_{i j}-u_{i l}\right) / h$, and the fact that $\max \left\{\left|u_{x}\right|,\left|u_{y}\right|\right\}$ $>|\nabla u| / \sqrt{2}$.

(ii) Writing

$$
H u_{i j}=\frac{u_{i+1, j}-2 u_{i j}+u_{i-1, j}}{h^{2}}+\frac{u_{i, j+1}-2 u_{i j}+u_{i, j-1}}{h^{2}}
$$

and expanding each term as a linear Taylor polynomial plus remainder, we obtain

$$
H u_{i j}=u_{x x}\left(\xi_{i}, y_{j}\right)+u_{y y}\left(x_{i}, \eta_{j}\right), \quad \xi_{i} \in\left(x_{i-1}, x_{i+1}\right), \eta_{j} \in\left(y_{j-1}, y_{j+1}\right) .
$$

Since the second derivative of $u$ is continuous on the compact set $\bar{\Omega}$, it is uniformly 
continuous, so that $\left\|H u_{i j}-\Delta u\left(x_{i}, y_{i}\right)\right\|_{\infty}=o(h)$ as $h \rightarrow 0$. The desired result follows.

(iii) If $q_{1}<0$, then $\Delta u>0$ throughout $\Omega_{1}$ and, for sufficiently small $h, q_{1}^{h}<0$ by (ii). For $q_{1}>0$, note that

$$
q_{1}^{h}=\left[\max _{\left(x_{i}, y_{j}\right) \in \Omega_{i}^{h}}\left(\frac{-\Delta u}{\max \left\{\left|u_{x}\right|,\left|u_{y}\right|\right\}}\right)\left(x_{i}, y_{j}\right)\right] \cdot[1+o(h)],
$$

which leads to the desired result.

(iv) If $q_{1}<0$, then by (iii), $\max \left\{1,1 /\left(1-h q_{1}^{h}\right)\right\}=1$ for $h$ sufficiently small, which is consistent with the desired bound. For the case $q_{1}>0$, (i) and (iii) imply that

$$
C_{1}^{h}(u)<\left(\frac{1}{1-h q_{1} \sqrt{2}}\right)^{\sqrt{2}[u] / h k_{1}}(1+o(h))<\exp \left(\frac{2 q_{1}[u]}{k_{1}}\right) \cdot(1+o(h)) .
$$

We are now ready to establish the basic stability and convergence result for the numerical scheme.

THEOREM 2. If $u \in C^{2}(\bar{\Omega})$ satisfies the condition (9), then, for $h$ sufficiently small, the discrete problem (10) has a unique solution $\left\{\alpha_{i j}\right\}$ assuming prescribed values on $\Gamma_{1}^{h}$. It satisfies the bound

$$
\left\|\alpha_{i j}\right\|_{\infty}<\left[C_{1}(u)\right]^{2} \cdot\left[\max \left\{\max _{\Gamma_{1}^{h}}\left|\alpha_{i j}\right|, \frac{\|f\|_{\infty}}{k_{2}}\right\}+\frac{2[u]}{k_{1}}\|f\|_{\infty}\right] \cdot(1+o(h)) .
$$

Moreover, if $u \in C^{3}(\bar{\Omega})$ and the solution $\alpha(x, y)$ of the continuous problem $L(\alpha ; u)=f$ has two continuous derivatives in $\bar{\Omega}$, then

$$
\left\|\alpha_{i j}-\alpha\left(x_{i}, y_{j}\right)\right\|_{\infty}=O(h) \text { as } h \rightarrow 0,
$$

assuming $\alpha_{i j}=\alpha\left(x_{i}, y_{j}\right)$ on $\Gamma_{1}^{h}$.

Proof The stability result follows from Theorem 1 and Lemma 6. The convergence rate can be established by noting that

$$
\mathcal{L}^{h}\left\{\alpha_{i j}-\alpha\left(x_{i}, y_{j}\right) ; u_{i j}\right\}=O(h),
$$

in view of (8) and the assumed differentiability in $\alpha$ and $u$, and then applying the stability result to (20).

Note that Theorem 2 can easily be tailored to accomodate the case where one or the other of $|\nabla u|$ or $\Delta u$ is uniformly positive throughout $\Omega$. For example, if $|\nabla u|>k_{1}>0$ in $\Omega$, we take $k_{2}=\infty$ and $\Omega_{2}=\Phi$ in (19), thus obtaining

$$
\left\|\alpha_{i j}\right\|_{\infty} \leqslant\left[C_{1}(u)\right]^{2} \cdot\left[\max _{\Gamma_{1}^{h}}\left|\alpha_{i j}\right|+\frac{2[u]}{k_{1}^{2}}\|f\|_{\infty}\right] \cdot[1+o(h)] .
$$

Likewise, if $\Delta u \geqslant k_{2}>0$ in $\Omega$, (19) becomes

$$
\left\|\alpha_{i j}\right\|_{\infty} \leqslant \max \left\{\max _{\Gamma_{1}^{h}}\left|\alpha_{i j}\right| \frac{\|f\|_{\infty}}{k_{2}}\right\} \cdot[1+o(h)] \text {. }
$$

4. Extensions of the Numerical Scheme. In this section, we extend the applicability of the difference scheme to irregular domains and to problems in which the basic condition (2) is violated but $\nabla u$ and $\Delta u$ do not simultaneously vanish anywhere in $\Omega$. 
We first consider the case of an irregular domain $\Omega$ which can be embedded in a larger domain $\Omega_{e}$ over which $u$ is defined and satisfies (2). We assume that $\operatorname{dist}\left\{\Omega, \Omega_{e}\right\}>0$ and superimpose a uniform grid over $\Omega_{e}$ (cf. Figure 2).

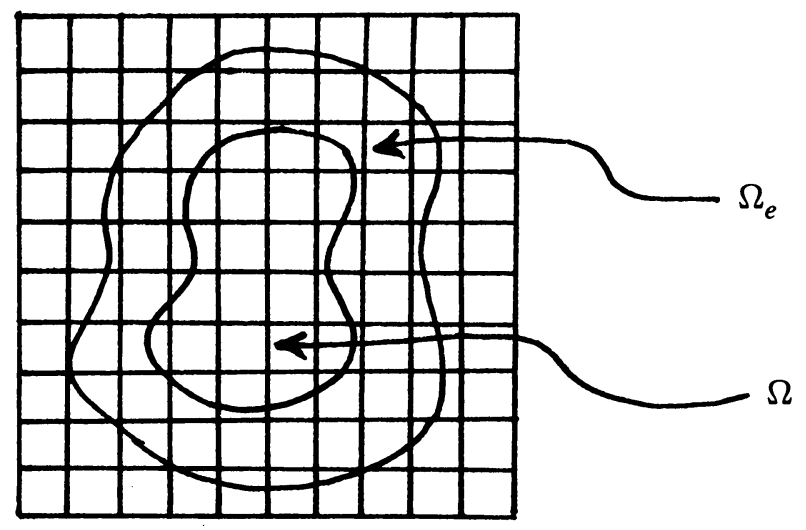

FigURE 2

As before, we denote the set of interior grid points $\left(x_{i}, y_{j}\right) \in \Omega$ by $\Omega^{h}$ and apply the difference scheme (3)-(5) for all $\left(x_{i}, y_{j}\right) \in \Omega^{h}$. For grid points of $\Omega^{h}$ lying adjacent to $\Gamma$, (3)-(5) will involve grid values of $u$ outside $\Omega$ but such values are well defined if $h<\operatorname{dist}\left\{\Omega, \Omega_{e}\right\}$, which we assume to be the case. Values of $\alpha$ outside $\Omega$ will also be required for the discretization at a point $\left(x_{i}, y_{j}\right) \in \Omega^{h}$ situated next to an inflow portion of $\Gamma$. Such a value, $\alpha_{i+1}$, say, can be approximated by the value of $\alpha$ at the intersection of the line segment from $\left(x_{i}, y_{j}\right)$ to $\left(x_{i+1}, y_{j}\right)$ with $\Gamma$. This introduces an error $O(h)$ if $\alpha \in C^{1}\left(\Omega_{e}\right)$.

Thus, the situation is essentially the same as before except that the "envelope" of $\Omega^{h}$ is no longer a square and the initial data has an $O(h)$ error. Theorems 1 and 2 are readily seen to retain their applicability, and we conclude that the modified numerical scheme produces $O(h)$ convergence provided $\alpha \in C^{2}\left(\Omega_{e}\right), u \in C^{3}\left(\Omega_{e}\right)$ and $u$ satisfies the basic condition (9) over $\Omega_{e}$.

We can also accomodate the case where values of $u$ outside $\Omega$ are not available. Here we define $\Gamma^{h}$ as the union of all points of intersection of the grid lines $x=x_{i}$, $y=y_{j}$ with $\Gamma$, and at each point $\left(x_{i}, y_{j}\right) \in \Omega^{h}$ we base the discretization of $L(\alpha ; u)=f$ on $\left(x_{i}, y_{j}\right)$ and its four nearest neighbors in the set $\Omega^{h} \cup \Gamma^{h}$. This necessitates minor changes in (3)-(5) to account for the nonuniformity in the grid adjacent to $\Gamma$, but the convergence rate is still $O(h)$.

We now indicate how the difference scheme can be applied to problems for which $u$ fails to satisfy (2). Suppose first that

$$
\inf _{\Omega}\{\max [|\nabla u|,-\Delta u]\}>0 .
$$

The difference scheme can then be applied to

$$
L(\alpha ; v)=-f, \quad v \equiv-u,
$$

where $v$ satisfies (2). Here the numerical scheme is propagated in the direction of decreasing $u_{i j}$, and $\alpha$ must be specified along the discrete outflow boundary. 
Next consider the more general situation where

$$
\inf _{\Omega}\{\max [|\nabla u|,|\Delta u|]\}>0 \text {. }
$$

Here $L(\alpha ; u)=f$ will not in general be uniquely solvable for $\alpha$ [7]. However, we may partition $\Omega$ into connected subdomains $\Omega^{(1)}, \Omega^{(2)}, \ldots$ such that for each $i$ either

$$
\inf _{\Omega^{(i)}}\{\max [|\nabla u|, \Delta u]\}>0 \text { or } \quad \inf _{\Omega^{(i)}}\{\max [|\nabla u|,-\Delta u]\}>0 .
$$

Assuming the availability of appropriate initial data, we may thus apply the numerical method locally within each $\Omega^{(i)}$ under the assurance that it will perform optimally. Thus, we have essentially covered all situations except that in which $\nabla u$ and $\Delta u$ vanish simultaneously somewhere in $\Omega$. This is an especially ill-conditioned case where $\|\alpha\|_{\infty}$ does not depend continuously on $\|f\|_{\infty}$ for the continuous problem $L(\alpha ; u)=f$ [7], and we omit consideration of it.

5. Test Conditions for Numerical Identification of the Diffusion Coefficient. In [7], we considered the following "test conditions" for observing a forward solution $u$ of the elliptic problem for $L(\alpha ; u)=f$ :

$$
\begin{aligned}
& \text { I. } f \geqslant f_{\min }>0 . \\
& \text { II. } u=0 \text { on } \Gamma \text {. }
\end{aligned}
$$

Assuming the underlying diffusion coefficient is positive, we showed that the resulting $u$ has the following properties:

(A) $|\nabla u| \geqslant \frac{\theta f_{\min }}{\|\nabla \alpha\|_{\infty}}$ and $/$ or $\Delta u \geqslant \frac{(1-\theta) f_{\min }}{\|\alpha\|_{\infty}}$ at each point of $\Omega$, any $\theta \in(0,1)$,
(B) $u<0$ in $\Omega$.

Property (A) insures that the basic condition (9) is satisfied, while (B) implies that the resulting hyperbolic problem $L(\alpha ; u)=f$ requires no Cauchy data. Here the characteristics of (1) originate at the minima of $u$ within $\Omega$, where $\alpha$ is given as in $\left(7^{\prime}\right)$.

We also impose the following regularity assumptions:

III. $\alpha \in C^{2}(\bar{\Omega}), f \in C^{1}(\bar{\Omega})$, and $\Omega$ satisfies the exterior sphere condition (for any $P \in \Gamma$ there exists a ball $B_{Q}$ centered at $Q \notin \bar{\Omega}$ such that $B_{q} \cap \bar{\Omega}=P$ ).

These regularity conditions insure (Gilbarg and Trudinger [4]) that

$$
u \in C^{3}(\Omega) \cap C^{0}(\bar{\Omega}) \text {. }
$$

Using properties (A), (B), (C), we now show that the numerical method performs optimally under the test conditions.

THEOREM 3. If a forward solution $u$ is observed under conditions I-III and the numerical scheme is applied to the resulting hyperbolic problem $L(\alpha u)=f$, no initial data for $\alpha$ is required for the discrete problem, and over any domain $\hat{\Omega}$, whose closure is contained within $\Omega$,

$$
\left\|\alpha_{i j}-\alpha\left(x_{i}, y_{j}\right)\right\|_{\infty}=O(h) \text { as } h \rightarrow 0 .
$$


Proof. Property (B) insures that $\Gamma_{1}^{h}=\Phi$, so that no initial data for $\alpha$ is required. For a given $\hat{\Omega}$, define

$$
\varepsilon=\sup _{\hat{\Omega}} u(<0), \quad \Omega_{\varepsilon}=\{x \in \Omega \mid u(x)<\varepsilon\} .
$$

Thus $\hat{\hat{\Omega}} \subset \Omega_{\varepsilon} \subset \Omega$ and $u \in C^{3}\left(\bar{\Omega}_{e}\right)$ by property (C). Moreover, $\alpha_{i j}$ values at grid points in $\Omega_{e}$ are not influenced by those in the remaining part of $\Omega$, since the latter are associated with higher $u$ values. Thus, we may apply Theorem 2 over $\Omega_{e}$ separately and conclude that $O(h)$ convergence ensues there, hence also in its subset $\hat{\Omega}$.

In the case of a $u$ produced by the test conditions, the difference scheme generates initial data for $\alpha$ via (7) at the relative minimum grid values of $u$ within $\Omega$. The solution is then propagated outward in the direction of increasing $u$, no initial data for $\alpha$ being required along $\Gamma$.

6. Some Test Computations. We list the results for two test problems over the unit square to which our numerical method has been applied. The forward solutions for the two problems are as depicted in Figures 3 and 4. In each case, $\alpha$ was chosen as $e^{-x y}$ and $f$ was obtained from $\alpha$ and $u$.

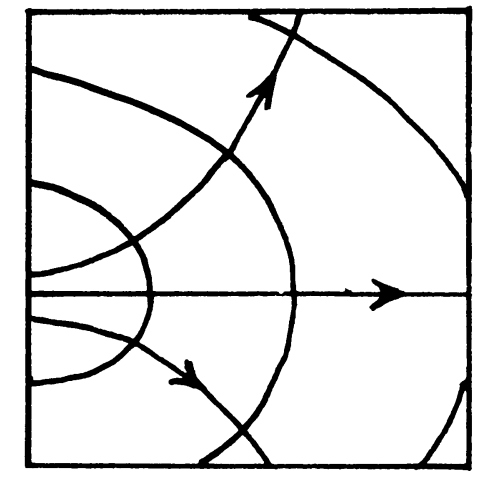

Figure 3

$$
u=(x+.2)^{2}+4(y-.4)^{2}
$$

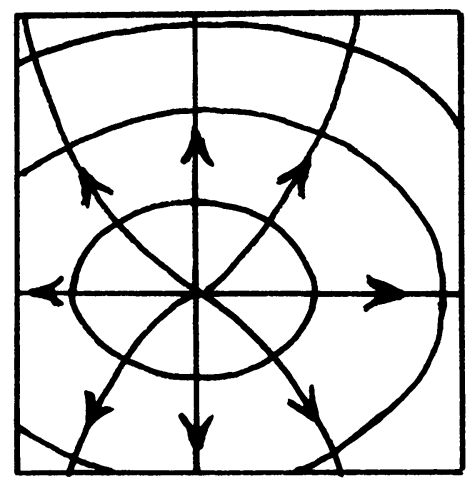

Figure 4

$u=(x-.3)^{2}+4(y-.4)^{2}$

In the first case, the discrete solution was developed from left to right and in opposite vertical directions away from the line $y=.4$ along each grid line $x=x_{i}$. Cauchy data for $\alpha$ was supplied along the line $x=0$. In the second case, the grid was scanned in an outward spiraling manner about the minimum grid value of $u$ and no initial data for $\alpha$ is required. (In accomodating more complicated configurations, a program which decided the scanning order internally would be of obvious value.) The computational results for the two cases are as follows, and the expected $O(h)$ convergence rate is apparent. 


\section{TABLE 1}

$\infty$-norm errors for test cases

$\begin{array}{rcc}n & \text { Case I } & \text { Case II } \\ 4 & .043 & .047 \\ 8 & .029 & .032 \\ 16 & .018 & .020 \\ 32 & .010 & .011 \\ 64 & .005 & .006\end{array}$

Acknowledgement. I thank Richard Falk for the many helpful discussions I had with him during the course of this research.

Department of Computer Science

Rutgers University

New Brunswick, New Jersey 08903

1. Jacob Bear, Dynamics of Fluids in Porous Media, American Elsevier, New York, 1972, pp. 214-215.

2. R. S. FALK, "Error estimates for the numerical identification of a variable coefficient," Math. Comp. (To appear.)

3. E. O. FRIND \& G. F. PINDER, "Galerkin solution of the inverse problem for aquifer transmissivity," Water Resour. Res., v. 9, 1973, pp. 1397-1410.

4. D. Gilbarg \& N. S. Trudnger, Elliptic Partial Differential Equations of Second Order, SpringerVerlag, Berlin and New York, 1977, pp. 101-106.

5. R. W. Nelson, "In-place determination of permeability distribution for heterogeneous porous media through analysis of energy dissipation," Soc. Pet. Eng. J., v. 8, 1968, pp. 33-42.

6. D. A. NuTBrown, "Identification of parameters in a linear equation of groundwater flow," Water Resour. Res., v. 11, 1975, pp. 581-588.

7. G. R. Richter, “An inverse problem for the steady state diffusion equation," SIAM J. Appl. Math. (To appear.) 